\title{
ESCALAS DE INTERESSES VOCACIONAIS (EIV): CONSTRUÇÃO, VALIDADE FATORIAL E CONSISTÊNCIA INTERNA'
}

\author{
Marco Antônio Pereira Teixeira* \\ Graciele Dotto Castro" \\ Carine Viegas Cavalheiro"
}

\begin{abstract}
RESUMO. O objetivo deste estudo foi construir um instrumento para avaliar seis dimensões de interesses vocacionais: realista (R), ivestigativa, atística (A), social (S), empreendedora (E) e cnvencional (C). Os itens gerados foram aplicados a estudantes de ensino médio e universitários, totalizando 857 protocolos válidos. Análises de componentes principais foram conduzidas para selecionar os melhores itens. Obteve-se uma solução de seis componentes, compatível com as expectativas teóricas, com 48 itens (8 por dimensão). A consistência interna (alpha de Cronbach) obtida para cada uma das escalas foi: $\mathrm{R}$ $(0,83)$, I $(0,79)$, A $(0,78), S(0,85)$, E $(0,74)$ e C $(0,80)$. Análises das subamostras de estudantes universitários e de ensino médio feitas separadamente produziram resultados similares. Comparações específicas entre pares contrastantes de cursos universitários revelaram a capacidade das escalas para discriminar grupos-critério; portanto, há evidências de que o instrumento proposto é válido e fidedigno, podendo ser utilizado em futuras pesquisas.
\end{abstract}

Palavras-chave: interesses profissionais, desenvolvimento vocacional, psicometria.

\section{VOCATIONAL INTERESTS SCALES (VIS): CONSTRUCTION, FACTORIAL VALIDITY AND INTERNAL CONSISTENCY}

\begin{abstract}
An instrument to assess six dimensions of vocational interests, namely, Realistic (R), Investigative (I), Artistic (A), Social (S), Enterprising (E) and Conventional (C) is established. Items generated to evaluate the proposed dimensions were applied to university and high school students, comprising 857 valid protocols. Principal components analysis was conducted to select the best items. A six component solution compatible with theoretical expectations was obtained for 48 items (6 for each dimension). Reliability estimates (Cronbach's alpha) for the scales were: R (0.83), I (0.79), A (0.78), S (0.85), E (0.74), and C (0.80). Separate analyses with university and high school students showed similar results. Mean comparison between contrasting pairs of university courses for each dimension showed that scales may discriminate criterion groups. Results indicate that the instrument is valid and reliable, and may be used in future research.
\end{abstract}

Key words: Vocational interests, vocational development, Psychometrics.

\section{ESCALAS DE INTERESES VOCACIONALES (EIV): CONSTRUCCIÓN, VALIDAD FACTORIAL Y CONSISTENCIA INTERNA}

RESUMEN. El objetivo de este estudio fue el construir un instrumento para avaluar seis dimensiones de intereses vocacionales: Realista (R), Investigativo (I), Artístico (A), Social (S), Emprendedor (E) y Convencional (C). Los ítemes generados fueron aplicados a estudiantes de secundaria y universitarios, totalizando 857 protocolos válidos. Análisis de componentes principales fueron conducidos para seleccionar los mejores ítemes. Se obtuvo una solución de seis componentes compatible con las expectativas teóricas con 48 ítemes (8 por dimensión). La consistencia interna (alpha de Cronbach) obtenida para cada una de las escalas fue: R (0,83), I $(0,79)$, A $(0,78), \mathrm{S}(0,85), \mathrm{E}(0,74)$ y C $(0,80)$. Análisis de las submuestras de estudiantes universitarios y de secundaria, por separado, produjeron resultados similares. Comparaciones específicas entre pares contrastantes de cursos universitarios revelaron la capacidad de las escalas para discriminar grupos-criterio. Por lo tanto, hay evidencias de que el instrumento propuesto es válido y fidedigno, pudiendo ser utilizado en futuras investigaciones.

Palabras-clave: intereses profesionales, desarrollo vocacional, psicometría.

Apoio: FAPERGS.

* Doutor em Psicologia. Docente no Instituto de Psicologia da Universidade Federal do Rio Grande do Sul-RS.

\# Psicóloga. 
Os interesses vocacionais são um construto importante no âmbito da psicologia de escolha e desenvolvimento de carreira. O termo interesse, contudo, possui múltiplos significados (Savickas, 1999). Em um sentido psicológico mais geral, o conceito de interesse refere-se à posição de um indivíduo em relação a um objeto específico, em termos da atenção que o objeto desperta no sujeito, do sentimento em relação ao objeto que acompanha a atenção (prazeroso/desprazeroso) e da tendência a aproximar-se ou não do objeto (querer ou não interagair com ele). Já quando usamos o termo interesses, especialmente no âmbito vocacional, referimo-nos a grupos de interesses específicos razoavelmente homogêneos e relacionados entre si, que constituem então uma disposição que tende a ser mais estável que um interesse particular. Assim, entendidos mais como traço do que como estado, os interesses denotam uma tendência de resposta razoavelmente estável, consistente e persistente, que aumenta a prontidão de um indivíduo para atender a certos tipos de estímulos ambientais e a agir em relação a eles (Savickas, 1999). Os interesses estão intimamente associados aos conceitos de necessidade e de valor. As necessidades são o impulso inicial para o comportamento, e emergem de um senso de incompletude ou falta que demanda satisfação. Os valores especificam os tipos de gratificação que poderão ser obtidos no ambiente em que o sujeito está inserido. Os interesses, por sua vez, revelam o modo como o indivíduo busca, no meio, realizar seus valores e atender às suas necessidades. Em comparação com as necessidades e os valores, os interesses são menos estáveis, pois eles dependem do contexto de estímulos ou objetos no qual o sujeito está inserido (Savickas, 1999). Assim, interesses vocacionais podem ser entendidos como disposições do indivíduo a envolverse em atividades ocupacionais que de algum modo contemplam valores e necessidades individuais.

A idéia de que os interesses vocacionais cumprem um papel importante na escolha de carreiras é bastante antiga, remontando ao século $\mathrm{XV}$, embora o primeiro esforço de sistematização teórica sobre o assunto date do início do século XX, com os trabalhos de Frank Parsons nos Estados Unidos da América do Norte. Para Parsons (1909), a escolha de uma carreira não deveria ser um evento que ocorresse ao acaso, e sim, um processo que contasse com o envolvimento ativo do sujeito na tarefa de escolha. Para tanto, cada indivíduo deveria conhecer suas habilidades e interesses de modo a procurar ocupações que combinassem com suas inclinações. O modelo proposto por Parsons foi posteriormente chamado de "modelo de traço e fator", devido à importância que atribuía às características individuais (traços) no processo de escolha (Brown \& Brooks, 1996).

Em 1959, John Holland propôs uma teoria de escolha vocacional baseada na idéia de traço e fator (Holland, 1959), a qual foi sendo ampliada e reformulada ao longo dos anos e constitui-se hoje em uma das mais pesquisadas no âmbito do desenvolvimento vocacional e de carreira (Spokane, 1996). A teoria de Holland mapeia seis principais dimensões de interesses vocacionais, que ele considera como expressões tanto da personalidade quanto dos próprios ambientes de trabalho. De fato, estudos empíricos têm apoiado a idéia de que as dimensões de interesses vocacionais propostas por Holland têm estreita relação com traços de personalidade (Holland, 1999). É importante ressaltar que o termo "vocacional" é utilizado aqui não em um sentido de uma característica individual que supostamente se encontre escondida nos indivíduos à espera de ser descoberta, mas apenas como um adjetivo que se refere àquilo que diz respeito ao mundo profissional ou ocupacional dos sujeitos.

De acordo com o modelo de Holland, uma escolha vocacional ótima resulta de uma combinação de características individuais com as características dos ambientes de trabalho. Ele postulou teoricamente, e empiricamente identificou seis grandes dimensões de interesses vocacionais e de ambientes de trabalho: realista, investigativa, artística, social, empreendedora e convencional (as iniciais de cada uma destas dimensões formam uma sigla pela qual o modelo também é conhecido: RIASEC). Segundo a teoria, as pessoas buscam ambientes nos quais possam exercer suas habilidades, expressar suas atitudes e valores e assumir papéis e problemas congruentes com seus interesses. Além disso, o comportamento individual não é determinado apenas pelas características pessoais ou do ambiente, mas resulta de uma interação entre personalidade e contexto (Spokane, 1996). Embora todas as pessoas apresentem nuanças das seis dimensões, o modelo de Holland postula a predominância de uma delas sobre as demais, caracterizando assim "tipos de personalidade" ou "personalidades vocacionais". As descrições dos tipos, no entanto, são apenas protótipos que caracterizam casos extremos; a maioria das pessoas apresenta uma mescla dos seis tipos do modelo. Os tipos prototípicos são descritos genericamente a seguir, de acordo com indicações de Holland (1997) e Spokane (1996).

Tipo realista: prefere trabalhar com dados objetivos, ao invés de suposições subjetivas. Valoriza coisas materiais e não costuma dar muita importância 
a sentimentos. Tende a ser quieto e reservado, mas com pouca auto-reflexão. Aprecia atividades práticas, nas quais possa ver um resultado mais imediato. Tende a ser conservador e autocontrolado. Em geral, demonstra interesse por atividades que envolvam manipulação de objetos, ferramentas, máquinas ou animais.

Tipo investigativo: aprecia trabalhar com o raciocínio, usando palavras ou idéias. Tende a ser analítico, racional, independente, introvertido, crítico, intelectual, inventivo, curioso, científico. Apresenta preferência por atividades que envolvem a investigação sistemática e criativa de fenômenos físicos, biológicos e culturais. Habilidades de pesquisa, mecânicas e aritméticas também podem caracterizar este tipo.

Tipo artístico: interage com o meio utilizando-se dos sentimentos, emoções, intuições e da imaginação. Tende a ser criativo, original, sonhador, idealista, rebelde, pouco convencional, às vezes descuidado. É sensível e expressivo, podendo ser também temperamental. Aprecia a estética e em geral não valoriza muito a conformidade, a responsabilidade e a lógica. Revela interesse por atividades livres e pouco sistematizadas que envolvam a manipulação de materiais físicos, verbais ou humanos para criar formas de arte ou produtos.

Tipo social: apresenta necessidade de interação social. Suas características são o entusiasmo, a amabilidade, a liderança, a persuasão, a sinceridade, a compreensão, a generosidade, o calor humano. Tende a ser extrovertido e cooperativo, embora possa eventualmente ser um tanto dependente dos demais. Busca ajudar as pessoas, muitas vezes sacrificando-se em favor dos outros. Demonstra interesse por atividades que envolvam o contato com outros para informar, treinar, desenvolver, curar ou educar.

Tipo empreendedor: busca agir sobre o mundo para conseguir o que deseja. Tende a ser aventureiro, entusiasta, dominante, extrovertido, impulsivo, persuasivo, sociável, versátil, ambicioso, líder, responsável, dinâmico, autoconfiante. Costuma valorizar assuntos de política, economia e negócios, e demonstra interesse por atividades em que possa comandar ou controlar aquilo que faz, buscando estabelecer contato com os outros para atingir objetivos organizacionais ou ganhos econômicos.

Tipo convencional: tende a se conformar às normas estabelecidas. Interage com o meio escolhendo metas, atitudes e valores que sejam aceitos pela sociedade. Suas características mais marcantes são o senso prático, a conformidade, o conservadorismo e a meticulosidade. Valoriza o trabalho metódico e a obediência, e em geral não se mostra muito criativo ou imaginativo. Prefere atividades que impliquem a manipulação sistemática e ordenada de dados, como guarda e organização de registros, preenchimento de documentos e uso de equipamentos de processamento de dados com o intuito de atingir suas metas.

Para Holland (1997), as dimensões do modelo apresentam um padrão de inter-relações que, num plano gráfico bidimensional, teria a forma de um hexágono, com os tipos aparecendo na seqüência $\mathrm{R}, \mathrm{I}$, A, S, E e C. Ou seja, as distâncias entre tipos adjacentes (RI, IA, AS, SE, EC, CR) seriam menores do que as distâncias observadas entre tipos alternados (RA, IS, AE, SC, ER, CI), que por sua vez seriam menores do que as distâncias entre tipos opostos (RS, IE, AC). Isto significa que as dimensões mais próximas entre si apresentam também maior semelhança quanto aos tipos a que se referem (por exemplo, o tipo I é mais próximo dos tipos $\mathrm{R}$ e $\mathrm{A}$ do que do $\mathrm{S}$, e mais distante do $\mathrm{E}$ e do $\mathrm{C}$ ).

Dada a sua formulação teórica clara e sua operacionalização através de instrumentos quantitativos bem validados (nos Estados Unidos), a teoria de Holland tem sido bastante aplicada no contexto do aconselhamento de carreira e dos estudos em desenvolvimento vocacional. O Self-Directed Search, desenvolvido por Holland, Fritzsche e Powell (1994), por exemplo, é um dos instrumentos mais utilizados em orientação profissional nos Estados Unidos. Além disso, o potencial explicativo da teoria vem suscitando diversas pesquisas. Sabe-se que os interesses vocacionais predizem a escolha de metas vocacionais e escolhas profissionais (Donnay \& Borgen, 1996; Hansen \& Sackett, 1993; Lent, Brown \& Hackett, 1994). Vários trabalhos, ainda, têm discutido a estrutura hexagonal dos interesses vocacionais (Day \& Rounds, 1998; Rounds \& Day, 1999; Rounds, McKenna, Hubert, \& Day, 2000; Tracey \& Rounds, 1995), o desenvolvimento dos interesses (Tracey, 2001, 2002), a relação entre interesses e habilidades (Ackerman \& Heggestad, 1997), interesses e lazer (Kerby \& Ragan, 2002), interesses e características de personalidade (Carson \& Mowsesian, 1993), além da aplicabilidade transcultural do modelo hexagonal (Einarsdóttir, Rounds, Ægisdóttir \& Gerstein, 2002; Farh, Leong \& Law, 1998; Glidden-Tracey \& Parraga, 1996; Rounds \& Tracey, 1996) - apenas para citar alguns exemplos das diversas áreas de pesquisa relacionadas à teoria de Holland.

Infelizmente, no Brasil, estudos empíricos metodologicamente adequados no campo da orientação profissional e do desenvolvimento 
vocacional ainda são incipientes. Em parte, isto se deve à escassez de instrumentos devidamente adaptados e validados em nossa cultura que permitam a realização de pesquisas. Em virtude de tal escassez de instrumentos validados na área de interesses vocacionais no Brasil, esta pesquisa teve os seguintes objetivos: a) construir um instrumento psicométrico de auto-relato para avaliar as dimensões de interesses vocacionais de acordo com o modelo RIASEC; b) obter evidências de validade fatorial e da capacidade discriminativa das escalas; e c) avaliar a fidedignidade das escalas obtidas.

\section{MÉTODO}

\section{Desenvolvimento dos itens do instrumento e estudo- piloto}

A partir das definições dos seis tipos de personalidades vocacionais propostas por Holland (1997), foram gerados pelos pesquisadores 113 itens para avaliar as seis dimensões, com cerca de 18 itens por dimensão. Optou-se, na elaboração do instrumento, por itens que se referissem a diferentes tipos de atividades ocupacionais, com graus variados de especificidade. Assim, o conteúdo de alguns itens criados foi associado à imagem de uma profissão específica (ou pelo menos o conteúdo dos itens sugeria uma associação mais direta com alguma atividade profissional), mas outros itens foram deliberadamente gerados de forma a não se associar exclusivamente a uma profissão. Isto foi feito para que a avaliação do nível de interesse dos sujeitos não ficasse restrita a atividades muito específicas, às quais os indivíduos poderiam apresentar rejeição em virtude de falta de conhecimentos acerca das profissões ou mesmo em função de uma visão muito estereotipada sobre tais atividades.

Os 113 itens produzidos foram então submetidos à avaliação de quatro juízes, que os classificaram de acordo com as definições de cada tipo (as definições foram fornecidas previamente aos juízes). Os itens que não foram classificados de acordo com o esperado (ou seja, correspondendo à dimensão para a qual foram construídos) por pelo menos três juízes tiveram o seu conteúdo revisado pelos pesquisadores ou foram eliminados (num total de 20 itens). Ao final, obteve-se um conjunto de 96 itens, com 16 para cada dimensão.

Os 96 itens foram a seguir analisados individualmente por quatro estudantes de ensino médio e seis estudantes universitários, que avaliaram a clareza dos itens. Nenhum item foi considerado de difícil compreensão pelos estudantes, embora em alguns tenha sido apontada certa ambigüidade de sentido. Estes itens foram então modificados para tornar o seu conteúdo semântico mais preciso.

Um estudo-piloto foi ainda realizado com uma amostra de 10 estudantes de ensino médio e 10 estudantes universitários, com o intuito de testar a compreensão destes quanto às instruções e ao uso do sistema de respostas.

A chave de respostas adotada foi elaborada em um sistema Likert de 7 pontos: -3 (me desagrada muito), -2 (me desagrada bastante), -1 (me desagrada um pouco), 0 (não me agrada nem me desagrada), +1 (me agrada um pouco), +2 (me agrada bastante) e +3 (me agrada muito). Os participantes do estudo-piloto não relataram dificuldades de compreensão quanto às instruções ou à forma de responder ao instrumento. Os itens foram ordenados em 16 blocos de 6 itens na sequiência realista, investigativo, artístico, social, empreendedor e convencional (por bloco).

\section{Participantes}

O instrumento foi aplicado a uma amostra total de 993 sujeitos, que produziram 857 protocolos válidos (completos). Destes participantes que preencheram o instrumento de forma completa, 468 eram estudantes de ensino médio (média de idade de 15,7 anos; deviopadrão de 1,29; 56,7\% mulheres) e 389 estudantes universitários (média de idade de 21,8 anos; desviopadrão de 4,22; 48,3\% mulheres).

\section{Instrumento}

O instrumento utilizado foi a versão com 96 itens, já incluindo modificações sugeridas pelos juízes e pelos sujeitos que fizeram a avaliação da clareza dos itens. Além das respostas aos itens, foi solicitado que os participantes indicassem o sexo, a idade e o curso (ou série) em que estavam matriculados.

\section{Procedimentos}

A aplicação do instrumento foi realizada coletivamente em salas de aula, após esclarecimentos sobre a natureza da pesquisa e a obtenção de consentimento informado. Para os estudantes menores de idade, foi solicitada também a autorização dos pais para participar da pesquisa.

\section{RESULTADOS}

\section{Análise da estrutura componencial do instrumento}

Um dos modos de verificar a validade de construto de um instrumento, ou seja, se ele representa 
adequadamente os construtos que se propõe a medir, consiste em empregar técnicas estatísticas de análise fatorial que indiquem a existência (ou não) das dimensões previstas teoricamente no conjunto de itens criado (Pasquali, 2004). Assim, os 96 itens foram submetidos a procedimentos exploratórios de análise de componentes principais, que é uma técnica estatística semelhante à análise fatorial. A primeira análise realizada apresentou um índice Kaiser-MeyerOlkin (KMO) de 0,90 e teste de esfericidade (Bartlett) com $p<0,001$, mostrando a adequação do conjunto dos dados para o procedimento. A análise indicou 21 componentes com eigenvalues maiores do que 1, e destes apenas 7 tinham valores maiores do que 2 . Contudo, uma análise visual do gráfico de scree sugeriu a presença de 6 componentes mais relevantes que explicaram $39,9 \%$ da variabilidade total, como se pode observar na Figura 1.

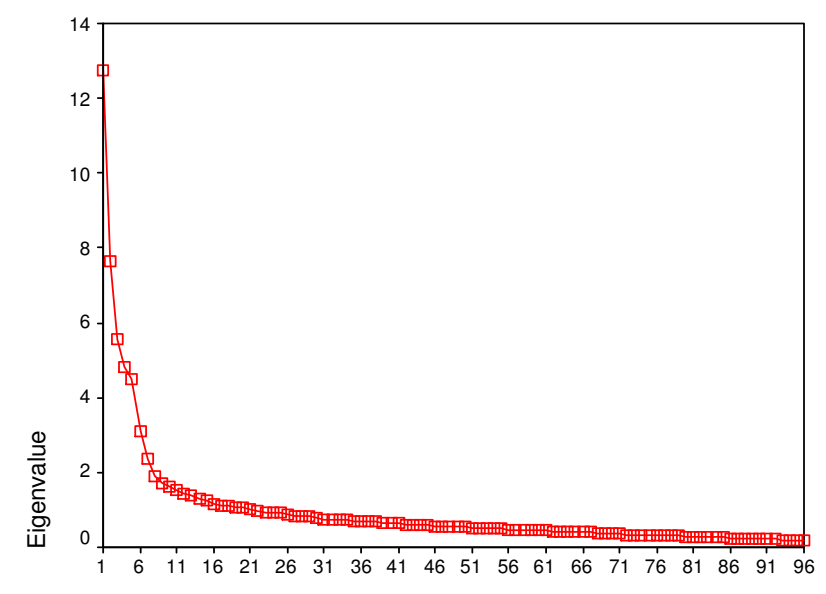

Número do componente

Figura 1. Gráfico de Scree com os Eigenvalues Obtidos na Primeira Análise de Componentes Principais.

Uma vez que as expectativas teóricas também previam a existência de seis dimensões subjacentes ao conjunto de itens, optou-se por reter os seis primeiros componentes e submetê-los a rotação oblíqua (já que se admite que as dimensões do modelo RIASEC estão correlacionadas). A análise da matriz das cargas componenciais indicou que a maioria dos itens apresentou cargas nos componentes conforme o esperado. Nesta primeira análise, foram considerados itens apropriados aqueles que apresentaram carga mais elevada no fator previsto e de valor igual ou superior a 0,30 . Os seguintes números de itens foram selecionados após este procedimento, por dimensão: 10 (realista), 10 (investigativo), 12 (artístico), 14 (social), 14 (empreendedor) e 11 (convencional).

A seguir, novas análises de componentes principais foram empreendidas com o intuito de selecionar itens que produzissem uma solução mais clara, ou seja, que tivessem cargas elevadas, preferencialmente, em um componente apenas. Além disso, buscou-se equalizar o número de itens nas diversas dimensões. Estabeleceu-se como critério básico para seleção dos itens um valor mínimo de carga componencial de 0,30 no componente esperado (embora se tenha admitido a possibilidade de um item ter cargas superiores a esse valor em outros componentes, desde que ocomponente previsto tivesse a carga mais alta). Como critério complementar, buscou-se reter itens que apresentassem variabilidade de conteúdo dentro de cada dimensão, evitando itens semelhantes.

Análises sucessivas permitiram chegar a uma solução claramente interpretável de seis dimensões, com oito itens mais relevantes por dimensão. O conjunto final de 48 itens foi analisado considerandose a amostra total (857 casos) e as subamostras de estudantes universitários (389 casos) e de ensino médio (468 casos). Nas três situações o gráfico de scree sugeriu a extração dos 6 primeiros componentes para a posterior rotação (oblíqua). Os índices KMO observados foram adequados (0,87 para a amostra total, 0,83 para a de estudantes universitários e 0,86 para a de estudantes de esnino médio), bem como os resultados do teste de esfericidade de Bartlett $(p s<0,001)$. Os percentuais totais de variância explicada pelos seis componentes extraídos foram, em cada caso: 47,2\% (total), 50,1\% (universitários) e $46,3 \%$ (ensino médio). O Anexo A exibe os itens da versão final do instrumento e os resultados da análise de componentes principais obtido com a amostra total (as soluções obtidas separadamente com os estudantes de ensino médio e universitários foram bastante semelhantes).

\section{Composição das escalas: estatísticas descritivas e avaliação da consistência interna}

Os oito itens mais relevantes de cada componente (como indicado na Tabela 1) foram tomados como escalas para avaliar as dimensões do modelo RIASEC. Assim, foram computados escores para cada uma das dimensões fazendo-se a média aritmética simples dos oito itens correspondentes a cada uma delas. A fidedignidade das escalas foi avaliada através do índice de consistência interna alpha de Cronbach. A Tabela 1 mostra as médias, desvios-padrão e coeficientes de fidedignidade observados, considerando-se a amostra total e as duas subamostras. Note-se que a amplitude máxima dos escores de cada escala pode variar entre -3 e +3 , o que corresponde à amplitude das respostas dadas a cada item. 
Tabela 1. Médias, Desvios-padrão e Índices de Fidedignidade.

\begin{tabular}{|c|c|c|c|c|c|c|c|c|c|}
\hline \multirow{3}{*}{ Escala } & \multicolumn{9}{|c|}{ Amostra } \\
\hline & \multicolumn{3}{|c|}{ Total } & \multicolumn{3}{|c|}{ Universitários } & \multicolumn{3}{|c|}{ Ensino médio } \\
\hline & alpha & $M$ & $D P$ & alpha & $M$ & $D P$ & Alpha & $M$ & $D P$ \\
\hline Realista & 0,83 & 0,36 & 1,25 & 0,84 & 0,32 & 1,25 & 0,83 & 0,39 & 1,25 \\
\hline Investigativo & 0,79 & 0,62 & 1,14 & 0,79 & 0,77 & 1,12 & 0,79 & 0,49 & 1,13 \\
\hline Artístico & 0,78 & 0,51 & 1,22 & 0,80 & 0,33 & 1,22 & 0,76 & 0,66 & 1,20 \\
\hline Social & 0,85 & 1,14 & 1,08 & 0,84 & 1,25 & 0,97 & 0,86 & 1,05 & 1,16 \\
\hline Empreendedor & r 0,76 & 1,08 & 0,97 & 0,80 & 1,15 & 0,98 & 0,72 & 1,03 & 0,96 \\
\hline Convencional & 0,80 & $-0,35$ & 1,17 & 0,79 & $-0,59$ & 1,12 & 0,79 & $-0,16$ & 1,17 \\
\hline
\end{tabular}

Evidências complementares de validade - análise por hipótese

A validade de construto de um instrumento também pode ser aferida através da testagem de hipóteses. Neste caso, pode-se verificar se o instrumento é capaz de discriminar um critério externo a ele mesmo, por exemplo, diferenciando grupos-critério que devem diferir no traço medido (Pasquali, 2004). Neste estudo, buscou-se colher evidências de validade para as escalas construídas verificando se elas seriam capazes de discriminar grupos de estudantes de diversos cursos superiores que, supostamente, deveriam apresentar diferenças, em média, em cada uma das dimensões do modelo. Uma vez que não foram localizados estudos brasileiros prévios mostrando diferenças entre alunos universitários no modelo RIASEC que pudessem ser tomados como parâmetro para a elaboração das hipóteses de diferenças, foi necessário elaborar um procedimento que indicasse quais cursos deveriam ser comparados entre si a fim de verificar a capacidade discriminativa das escalas. Inicialmente, os pesquisadores elaboraram uma lista de pares de cursos contrastantes para cada uma das dimensões, com base na descrição teórica dos tipos e em uma interpretação subjetiva dos estereótipos profissionais em nossa cultura. A seguir, estes pares contrastantes foram submetidos à avaliação de 13 juízes (estudantes de psicologia), que julgaram, com base em suas percepções pessoais e nas definições teóricas dos tipos, se deveria haver diferenças, em média, entre os pares de grupos propostos. Foram selecionados para a análise estatística posterior apenas os pares de cursos que obtiveram concordância quanto ao contraste igual ou superior a $80 \%$ (quando mais de um par para um mesmo tipo teve concordância superior ao especificado, optou-se pelo par com o maior índice de concordância). A expectativa era encontrar diferenças estatisticamente significativas nas médias dos grupos comparados. A Tabela 2 exibe os pares contrastantes selecionados para cada uma das dimensões de interesse vocacional, assim como as médias, desvios-padrão e resultados dos testes t para comparação de médias.
Tabela 2. Médias, Desvios-padrão e Testes $t$ para Grupos Contrastantes.

\begin{tabular}{llllll}
\hline & $\boldsymbol{M}$ & $\boldsymbol{D P}$ & $\boldsymbol{n}$ & $\boldsymbol{T}$ & $\boldsymbol{p}$ \\
\hline Realista & & & & & \\
$\quad$ Química industrial & 0,25 & 1,04 & 8 & 2,11 & 0,039 \\
$\quad$ Ciências sociais & $-0,40$ & 1,37 & 16 & & \\
Investigativo & & & & & \\
$\quad$ Física & 1,15 & 0,79 & 22 & 2,76 & 0,009 \\
$\quad$ Administração & 0,38 & 1,05 & 21 & & \\
Artístico & & & & & \\
$\quad$ Arquitetura & 1,14 & 0,98 & 20 & 2,34 & 0,025 \\
$\quad$ Fonoaudiologia & 0,26 & 1,37 & 21 & & \\
$\quad$ Social & & & & & \\
$\quad$ Fonoaudiologia & 2,37 & 0,47 & 21 & 6,02 & 0,001 \\
$\quad$ Química industrial & 0,89 & 1,07 & 32 & & \\
Empreendedor & & & & & \\
$\quad$ Administração & 1,85 & 0,96 & 21 & 3,72 & 0,001 \\
$\quad$ Odontologia & 0,80 & 0,85 & 21 & & \\
Convencional & & & & & \\
$\quad$ Direito & $-0,27$ & 0,94 & 56 & 3,78 & 0,001 \\
$\quad$ Desenho industrial & $-1,27$ & 0,87 & 16 & & \\
\hline
\end{tabular}

Como se pode observar, em todas as comparações realizadas as diferenças observadas foram estatisticamente significativas, indicando que as escalas são capazes de discriminar grupos que, supostamente, diferem em cada uma das dimensões de interesse, o que sugere a validade do instrumento.

\section{DISCUSSÃO}

Os resultados obtidos permitem-nos concluir que a presente pesquisa atingiu seu objetivo principal, que foi a construção de um instrumento de autorelato para avaliar as dimensões de interesses vocacionais de acordo com o modelo RIASEC. As análises de componentes principais mostraram que as seis dimensões previstas teoricamente emergiram empiricamente dos dados, conferindo validade fatorial ao instrumento proposto. Vale ressaltar que as soluções obtidas nas análises dos componentes principais foram muito nítidas, tanto para a amostra total quanto para as subamostras de estudantes universitários e de ensino médio.

Da mesma forma, os índices de consistência interna obtidos também foram altamente satisfatórios, especialmente se levarmos em consideração o pequeno número de itens em cada escala (apenas oito). Dos 18 índices alpha calculados (considerando a amostra total e as duas subamostras), nove foram iguais ou superiores a 0,80 , oito situaram-se entre 0,76 e 0,79 e apenas um foi inferior a 0,75 (no caso, 0,72). Estes resultados são muito positivos e indicam que as escalas 
propostas podem ser usadas, no âmbito de pesquisas, como medidas fidedignas dos seus respectivos construtos.

Em relação às evidências complementares de validade das escalas, ou seja, sua capacidade de discriminar grupos entre os quais se esperariam diferenças significativas, os resultados também foram favoráveis. Em todas as comparações feitas as escalas mostraram-se capazes de apontar diferenças significativas entre os grupos contrastantes conforme esperado, o que sugere sua validade.

Em resumo, o presente estudo teve por objetivo construir um instrumento de auto-relato para avaliar dimensões de interesse vocacional dentro do modelo RIASEC, além de obter evidências acerca de sua validade e fidedignidade. Os resultados obtidos indicam, em seu conjunto, o sucesso do empreendimento. As dimensões previstas emergiram das análises de componentes principais, evidenciando a validade fatorial do instrumento. Em complemento, as escalas mostraram-se capazes de detectar diferenças significativas entre grupos selecionados, o que é mais um indicador da validade do instrumento. Por sua vez, os índices de fidedignidade observados para as escalas também variaram de satisfatórios a excelentes. De modo geral, conclui-se que o instrumento proposto pode ser empregado em futuras pesquisas que pretendam avaliar as dimensões do modelo RIASEC, ao menos em populações de estudantes de ensino médio e de universitários. Pesquisas complementares são ainda necessárias a fim de verificar a validade e a fidedignidade do instrumento em populações diferentes das que foram aqui estudadas. Por fim, deve-se salientar que aplicações clínicas do instrumento não são recomendadas, uma vez que os resultados desta pesquisa não se constituem em normas que possam ser utilizadas para efeitos de avaliação individual, além de serem necessários outros estudos que investiguem de modo mais detalhado a aplicabilidade do modelo RIASEC em nossa cultura.

\section{REFERÊNCIAS}

Ackerman, P. L. \& Heggestad, E. D. (1997). Intelligence, personality, and interests: Evidence for overlapping traits. Psychological Bulletin, 121, 219-245.

Brown, D. \& Brooks, L. (1996, $3^{\text {a }}$ ed.). Introduction to theories of career development and choice: Origins, evolution, and current efforts. In D. Brown \& L. Brooks (Eds.), Career choice and development (pp. 1-32). San Francisco: Jossey-Bass.
Carson, A. D. \& Mowsesian, R. (1993). Self-monitoring and private self-consciousness: Relations to Holland's personality types. Journal of Vocational Behavior, 42, 212-222.

Day, S. X. \& Rounds, J. (1998). Universality of vocational interest structure among racial and ethnic minorities. American Psychologist, 53, 728-736.

Donnay, D. A. C. \& Borgen, F. H. (1996). Validity, structure and content of the 1994 Strong Interest Inventory. Journal of Counseling Psychology, 43, 275-291.

Einarsdóttir, S., Rounds, J., Ægisdóttir, S. \& Gerstein, L. H. (2002). The structure of vocational interests in Iceland: Examining Holland's and Gati's RIASEC models. European Journal of Psychological Assessment, 18, 8595.

Farh, J., Leong, F. T. L. \& Law, K. S. (1998). Cross-cultural validity of Holland's model in Hong Kong. Journal of Vocational Behavior, 52, 425-440.

Glidden-Tracey, C. E. \& Parraga, M. I. (1996). Assessing the structure of vocational interests among bolivian university students. Journal of Vocational Behavior, 48, 96-106.

Hansen, J. C. \& Sackett, S. A. (1993). Agreement between college major and vocational interests for female athlete and non-athlete college students. Journal of Vocational Behavior, 43, 298-309.

Holland, J. G. (1959). A theory of vocational choice. Journal of Counseling Psychology, 6, 35-45.

Holland, J. H. (1997, $3^{\text {a }}$ Ed.). Making vocational choices: A theory of vocational personalities and work environments. Odessa: Psychological Assessment Resources.

Holland, J. H. (1999). Why interest inventories are also personality inventories. In M. L. Savickas \& A. R. Spokane (Eds.), Meaning, mesasurement and counseling use of vocational interests (pp. 87-101). Palo Alto: Davies-Black.

Holland, J., Fritzsche, B. \& Powell, A. (1994). Technical manual for the Self-Directed Search. Lutz: Psychological Assessment Resources.

Kerby, D. S. \& Ragan, K. M. (2002). Activities interests and Holland's RIASEC system in older adults. International Journal of Aging and Human Development, 55, 117-139.

Lent, R. W., Brown, S. D. \& Hackett, G. (1994). Toward a unifying social cognitive theory of career and academic interest, choice, and performance. Journal of Vocational Behavior, 45, 79-122.

Parsons, F (1909). Choosing a vocation. Boston: Houghton Mifflin.

Pasquali, L. (2004). Psicometria: teoria dos testes na psicologia e na educação. Petrópolis: Vozes.

Rounds, J. \& Day, S. X. (1999). Describing, evaluating, and creating vocational interest structures. In M. L. Savickas \& A. R. Spokane (Eds.), Meaning, mesasurement and counseling use of vocational interests (pp. 103-133). Palo Alto: Davies-Black. 
Rounds, J. \& Tracey, T. J. (1996). Cross-cultural structural equivalence of RIASEC models and measures. Journal of Counseling Psychology, 43, 310-329.

Rounds, J., McKenna, M. C., Hubert, L. \& Day, S. X. (2000). Tinsley on Holland: A misshapen argument. Journal of Vocational Behavior, 56, 205-215.

Savickas, M. L. (1999). The psychology of interests. In M. L. Savickas \& A. R. Spokane (Eds.), Meaning, mesasurement and counseling use of vocational interests (pp. 19-56). Palo Alto: Davies-Black.

Spokane, A. (1996, 3ª ed.). Holland's theory. In D. Brown \& L. Brooks (Eds.), Career choice and development (pp. 33-74). San Francisco: Jossey-Bass.
Tracey, T. J. (2002). Development of interests and competency beliefs: A 1-year longitudinal study of fifth to eight grade students using the ICA-R and structural equation modeling. Journal of Counseling Psychology, 49, 148-163.

Tracey, T. J. G. \& Rounds, J. (1995). The arbitrary nature of Holland's RIASEC types: A concentric-circles structure. Journal of Counseling Psychology, 42, 431-439.

Recebido em 16/01/06 Aceito em 08/01/07

Endereço para correspondência: Tarco Antônio Pereira Teixeira. Rua Ramiro Barcelos, 2600, Instituto de Psicologia da UFRGS, CEP 900035-003, Porto Alegre-RS. E-mail: mapteixeira@yahoo.com.br 